\title{
Radiation-Induced Esophagitis Exacerbated by Everolimus
}

\author{
Yuji Miura ${ }^{a}$ Koichi Suyama $^{a} \quad$ Akihiko Shimomura $^{a} \quad$ Jimpei Miyakawab \\ Hiroki Kobayashic Akiyoshi Uki ${ }^{c}$ Toshikazu Okaneya ${ }^{b}$ Toshimi Takano $^{a}$ \\ Departments of ${ }^{a}$ Medical Oncology, ${ }^{b}$ Radiology and ' Urology, Toranomon Hospital, \\ Tokyo, Japan
}

\section{Key Words}

Mammalian target of rapamycin $\cdot$ Renal cell carcinoma $\cdot$ Radiation recall syndrome

\begin{abstract}
Background: Everolimus, a potent mammalian target of rapamycin (mTOR) inhibitor, has shown anticancer activity against various types of cancer, including renal cell carcinoma (RCC); however, little information is available on the efficacy and safety of the combination of everolimus and radiotherapy. We report a case of radiation-induced esophagitis that might have been exacerbated by the sequential administration of everolimus. Case Presentation: A 63-year-old Japanese man with RCC complained of back pain, and magnetic resonance imaging revealed vertebral metastases. He received radiotherapy (30 Gy/10 fractions) to the T6-10 vertebrae. Everolimus was administered immediately after the completion of radiotherapy. One week later, he complained of dysphagia, nausea and vomiting. An endoscopic examination of the esophagus showed erosive esophagitis in the middle to lower portions of his thoracic esophagus, corresponding to the irradiation field. Conclusion: Clinicians should be aware that everolimus might lead to the unexpected exacerbation of radiation toxicities.
\end{abstract}

\section{Background}

Renal cell carcinoma (RCC) accounts for $80-85 \%$ of all primary kidney neoplasms [1]. There are approximately 65,000 new cases of RCC and 14,000 deaths from RCC each year in the USA [2]. Twenty-five to $30 \%$ of patients newly diagnosed with RCC have metastases at initial presentation, and $20-30 \%$ of patients with localized RCC experience relapse after 
radical nephrectomy over the course of several years of follow-up [1]. Frequent sites of metastasis include the lung, bone and liver.

Recently, advances in the understanding of the biology of RCC have led to the development of targeted agents such as vascular endothelial growth factor receptor inhibitors and mammalian target of rapamycin (mTOR) inhibitors. Patients with metastatic RCC or those who develop recurrent metastases following prior treatment generally receive systemic chemotherapy using these molecular targeted agents. Meanwhile, treatment of symptomatic metastases includes radiotherapy or surgical procedures. External beam radiotherapy is one of the standard palliative treatments for painful bone metastasis, which is the second most common site of metastasis in RCC. However, it is unknown whether the continual administration of molecular targeted agents during palliative radiotherapy is safe or not. Everolimus, a potent mTOR inhibitor, has shown anticancer activity against various types of cancer, including RCC. Some preclinical studies have shown that mTOR inhibitors are potential radiosensitizers [3]. However, little information is available on the clinical efficacy or safety of the combination of mTOR inhibitors and radiotherapy.

We report a case of radiation-induced esophagitis that might have been exacerbated by the sequential administration of everolimus.

\section{Case Presentation}

A 63-year-old man was diagnosed with RCC (papillary cell carcinoma) and underwent a nephrectomy in December 2006. Four years later, lung metastases were found and a lung metastasectomy was performed. However, the lung metastases recurred in June 2011, and sunitinib therapy was initiated. The lung metastases improved, and the sunitinib treatment was continued for 12 months. In July 2012, the patient complained of back pain, and magnetic resonance imaging and a technetium bone scintigraphy examination revealed multiple vertebral metastases. Treatment with everolimus (10 mg daily) was initiated as second-line systemic therapy, and nonsteroidal anti-inflammatory drugs as well as oxycodone analgesics were also administered. One week later, the patient's back pain persisted, and we temporarily discontinued the everolimus treatment and initiated irradiation to the T6-10 vertebrae at 30 Gy in 10 fractions over a 2-week period. Everolimus (10 mg daily) was reinitiated immediately after the completion of the radiotherapy. One week after receiving the everolimus, the patient complained of dysphagia, nausea and vomiting. An endoscopic examination of the esophagus showed erosive esophagitis in the middle to lower portions of his thoracic esophagus (fig. 1), corresponding to the irradiation field (fig. 2a, b). Everolimus was discontinued, and his symptoms gradually resolved. One month later, a repeat endoscopy showed a remarkable improvement in the esophageal erosions (fig. 3).

Written informed consent was obtained from the patient for publication of this case report and any accompanying images. A copy of the written consent form is available for review by the editor of this journal.

\section{Discussion}

This case suggests that everolimus administration might exacerbate or augment radiation toxicity. Generally, a radiation dose of 30 Gy delivered in 10 fractions would rarely cause 
Miura et al.: Radiation-Induced Esophagitis Exacerbated by Everolimus

radiation-induced esophagitis. Several hypotheses can be proposed concerning the pathogenesis of severe esophagitis associated with everolimus and radiotherapy.

First, the administration of everolimus immediately after radiation might contribute to the development of radiation recall syndrome. Radiation recall syndrome is an inflammatory reaction that occurs when certain promoting agents are administered during or soon after radiotherapy [4]. The biologic basis of this syndrome is unknown. Although it most commonly develops in the skin, other sites such as the gastrointestinal tract (esophagus, small intestine, etc.) and lung have also been reported [5]. Radiation recall syndrome has been associated with various drugs, including everolimus [5, 6]. A previous study reported that 3 patients who received an mTOR inhibitor (everolimus or temsirolimus) developed gastritis, bladder stenosis and colitis related to radiation recall syndrome [6]. In that study, the time interval between the completion of radiotherapy and the recall reaction ranged from 2 months to 4 years [6]. However, the 1-week interval in the present case differs from that described in previous reports.

Second, everolimus might contribute to enhanced radiation toxicity. Preclinical data have suggested the synergic effects of mTOR inhibitors when combined with radiation $[7,8]$. One mechanism for enhancing the antitumor effect is thought to be an increase in antiangiogenesis efficacy when mTOR inhibitors are combined with radiotherapy. Shinohara et al. [8] reported that everolimus combined with radiation significantly reduced the tumor vasculature in a glioma xenograft. Another mechanism of radiosensitization is the inhibition of DNA double-strand break repair by mTOR inhibitors. Chen et al. [9] have demonstrated that rapamycin combined with ionizing radiation inhibited homologous recombination and nonhomologous end joining, two major pathways for the repair of radiation-induced DNA double-strand breaks. These mechanisms are consistent with the exacerbation of radiation toxicity induced by mTOR inhibitors. The two major mechanisms of radiation-induced mucositis are the impairment of vascular endothelial cells and DNA strand breaks, leading to cell death in the basal layer of the epithelium.

Some clinical data have shown the efficacy of concurrent mTOR inhibitors and radiotherapy; however, little information is available on the toxicity of concurrent radiation and mTOR inhibitors $[10,11]$.

\section{Conclusion}

Little clinical evidence regarding the safety of mTOR inhibitors when used in combination with radiation has been reported. Clinicians should be aware that such combinations might lead to the unexpected exacerbation of radiation toxicities. Further clinical studies on the safety and efficacy of radiotherapy combined with mTOR inhibitors are warranted.

\section{Acknowledgement}

The authors thank Dr. Richard L. Theriault (Department of Breast Medical Oncology, University of Texas, MD Anderson Cancer Center) and the Japan TeamOncology Program for their educational programs. 


\section{Disclosure Statement}

The authors declare that they have no competing interests.

\section{References}

Motzer RJ, Bander NH, Nanus DM: Renal-cell carcinoma. N Engl J Med 1996;335:865-875. Siegel R, Naishadham D, Jemal A: Cancer statistics, 2013. CA Cancer J Clin 2013;63:11-30.

-3 Murphy JD, Spalding AC, Somnay YR, Markwart S, Ray ME, Hamstra DA: Inhibition of mTOR radiosensitizes soft tissue sarcoma and tumor vasculature. Clin Cancer Res 2009;15:589-596.

$\checkmark 4$ Caloglu M, Yurut-Caloglu V, Cosar-Alas R, Saynak M, Karagol H, Uzal C: An ambiguous phenomenon of radiation and drugs: recall reactions. Onkologie 2007;30:209-214.

$\checkmark 5$ Azria D, Magne N, Zouhair A, Castadot P, Culine S, Ychou M, Stupp R, Van Houtte P, Dubois JB, Ozsahin M: Radiation recall: a well recognized but neglected phenomenon. Cancer Treat Rev 2005;31:555-570.

-6 Bourgier C, Massard C, Moldovan C, Soria JC, Deutsch E: Total recall of radiotherapy with mTOR inhibitors: a novel and potentially frequent side-effect? Ann Oncol 2011;22:485-486.

7 Eshleman JS, Carlson BL, Mladek AC, Kastner BD, Shide KL, Sarkaria JN: Inhibition of the mammalian target of rapamycin sensitizes U87 xenografts to fractionated radiation therapy. Cancer Res 2002;62:7291-7297.

$\checkmark 8$ Shinohara ET, Cao C, Niermann K, Mu Y, Zeng F, Hallahan DE, Lu B: Enhanced radiation damage of tumor vasculature by mTOR inhibitors. Oncogene 2005;24:5414-5422.

-9 Chen H, Ma Z, Vanderwaal RP, Feng Z, Gonzalez-Suarez I, Wang S, Zhang J, Roti Roti JL, Gonzalo S, Zhang J: The mTOR inhibitor rapamycin suppresses DNA double-strand break repair. Radiat Res 2011;175:214-224.

-10 Sarkaria JN, Galanis E, Wu W, Dietz AB, Kaufmann TJ, Gustafson MP, Brown PD, Uhm JH, Rao RD, Doyle L, et al: Combination of temsirolimus (CCI-779) with chemoradiation in newly diagnosed glioblastoma multiforme (GBM) (NCCTG trial N027D) is associated with increased infectious risks. Clin Cancer Res 2010;16:5573-5580.

11 Sarkaria JN, Galanis E, Wu W, Peller PJ, Giannini C, Brown PD, Uhm JH, McGraw S, Jaeckle KA, Buckner JC: North Central Cancer Treatment Group Phase I trial N057K of everolimus (RAD001) and temozolomide in combination with radiation therapy in patients with newly diagnosed glioblastoma multiforme. Int J Radiat Oncol Biol Phys 2011;81:468-475.

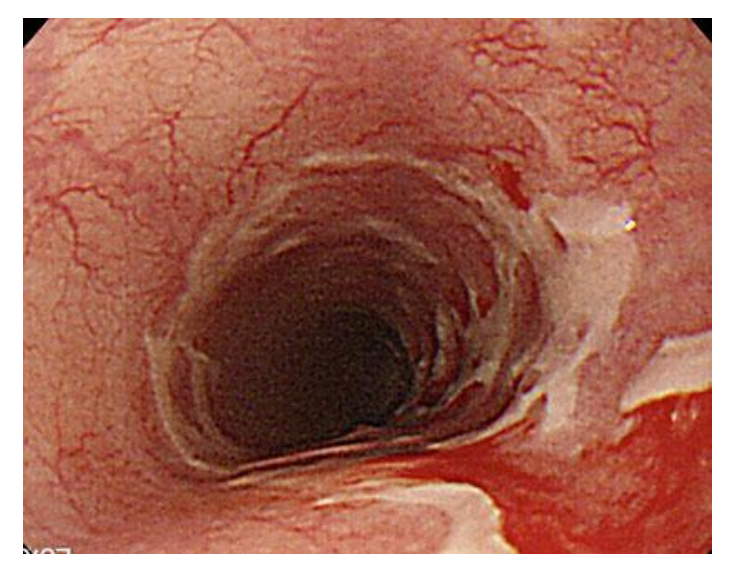

Fig. 1. Radiation-induced esophagitis. Endoscopy examination reveals erosion and erythema in the middle to lower portions of the thoracic esophagus. 


\section{Case Reports in Oncology}

\begin{tabular}{l|l}
\hline Case Rep Oncol 2013;6:320-324 & \\
\hline DOI: $10.1159 / 000353309$ & $\begin{array}{l}\text { C 2013 S. Karger AG, Basel } \\
\text { www.karger.com/cro }\end{array}$ \\
\hline
\end{tabular}

Miura et al.: Radiation-Induced Esophagitis Exacerbated by Everolimus

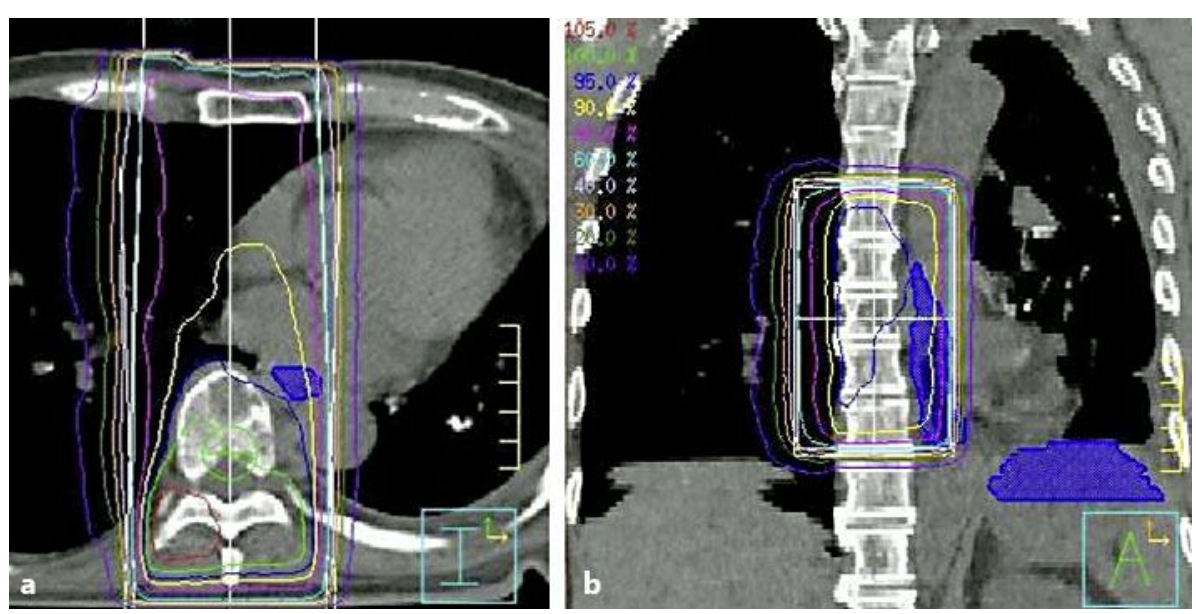

Fig. 2. Irradiation field for vertebral metastases (T6-10). The irradiated region for vertebral metastases (T6-10) is shown in the horizontal (a) and coronal (b) sections. Blue areas show the esophagus and upper stomach.

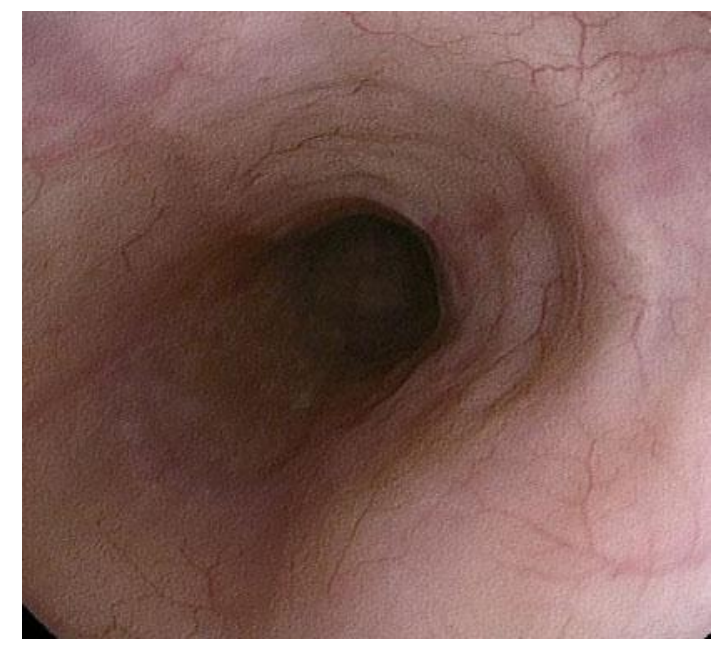

Fig. 3. Repeat endoscopy 1 month after discontinuation of everolimus shows a remarkable improvement in the esophageal erosions. 